\title{
Review on Machine Translation Post-Editing of Science and Technology Texts in China
}

\author{
Huijun Ren1, Jingxuan Wang1, Jingya Pang ${ }^{1}$, Luna $W^{1}{ }^{1}$, Junhua Shi ${ }^{2}$ \\ ${ }^{1}$ School of Arts and Sciences, Shaanxi University of Science and Technology, Xi'an, China \\ ${ }^{2}$ School of Marxism, Shaanxi University of Science and Technology, Xi'an, China \\ Email: renhj@sust.edu.cn
}

How to cite this paper: Ren, H. J., Wang, J. X., Pang, J. Y., Wu, L. N., \& Shi, J. H. (2020). Review on Machine Translation PostEditing of Science and Technology Texts in China. Open Journal of Modern Linguistics, 10, 1-10.

https://doi.org/10.4236/ojml.2020.101001

Received: December 27, 2019

Accepted: January 12, 2020

Published: January 15, 2020

Copyright $\odot 2020$ by author(s) and Scientific Research Publishing Inc. This work is licensed under the Creative Commons Attribution International License (CC BY 4.0).

http://creativecommons.org/licenses/by/4.0/

\begin{abstract}
Nowadays, with the popularization of machine translation software, new progress has been made in the development of machine translation post-editing of science and technology texts. However, researches on science and technology text post-editing in China has not been developed correspondingly. This paper collates and summarizes all the literatures on science and technology text post-editing in China, makes them into four categories based on the type of source texts and provides the corresponding features and analyses respectively, sorts out three major problems of current researches in this field, and points out targeted future research directions, so as to make a detailed analysis of problems and promote progress in this field.
\end{abstract}

\section{Keywords}

Science and Technology Text, Post-Editing, Machine Translation

\section{Introduction}

With the increasingly development of global science and technology and the wider research and application of machine translation software, the machine translation post-editing (MTPE) also arises at the historic moment. According to the 2010 language services industry report released by Common Sense Advisory (CSA), $42 \%$ of language service providers have provided machine translation post editing services to customers (DePalma \& Hegde, 2010). MTPE refers to the process of processing and modifying the source output of machine translation with a certain purpose (Feng \& Cui, 2016). China's research on MTPE starts relatively late. Inputting "post-editing" as the key word to search literatures in CNKI (the most authorative academic website in China), the number of relevant papers was only 78 . The earliest MTPE research in China was about intelligent 
post-editing machine by Huang Heyan and other scholars in 1995. There are even less researches on MTPE of science and technology texts. Of all these papers, only 16 papers discuss or mention science and technology texts. The paper Analyzing the Post-editing of Machine Translation at Autodesk does not discuss post-editing except that the source text belongs to a science and technology text which discusses MTPE. The paper The Critial Application of Machine Translation in Translation Practice-A Case of Search Engine Marketing, Inc. Translation Program (Fu, 2016) talks about the quality of MTPE, but the source text Search Engine Marketing belongs to a business text, not a science and technology text, therefore, the paper will not discuss these two papers. According to the different types of science and technology texts, this paper sorts out and summarizes the rest 14 papers.

\section{Review on Four Classifications}

Liu Miqing gave the definition of science and technology text in his book English Varieties and Translation as the written and spoken language of all contents in the field of science and technology, including scientific and technological writings, papers and reports, experimental reports, programs, various kinds of scientific and technological information, written materials, user manuals and guides, as well as scientific and technological talks, scientific and technological meetings, films, videos, etc. (Liu, 1998). According to this definition, the paper divides the source text of all the 14 papers into four categories: scientific and technological writings (STW), user manuals (UM), text materials (TM) and video and audio (AA), as shown in Figure 1.

Classified by the type of the those 14 source texts, there are 7 papers of scientific and technological writings, 3 papers of user manuals, 2 papers of text materials, and 1 paper of video and audio. Another 1 paper belongs to the category of training science and technology text post-editing translator; therefore, it is not divided into these four categories. According to the above classification, these

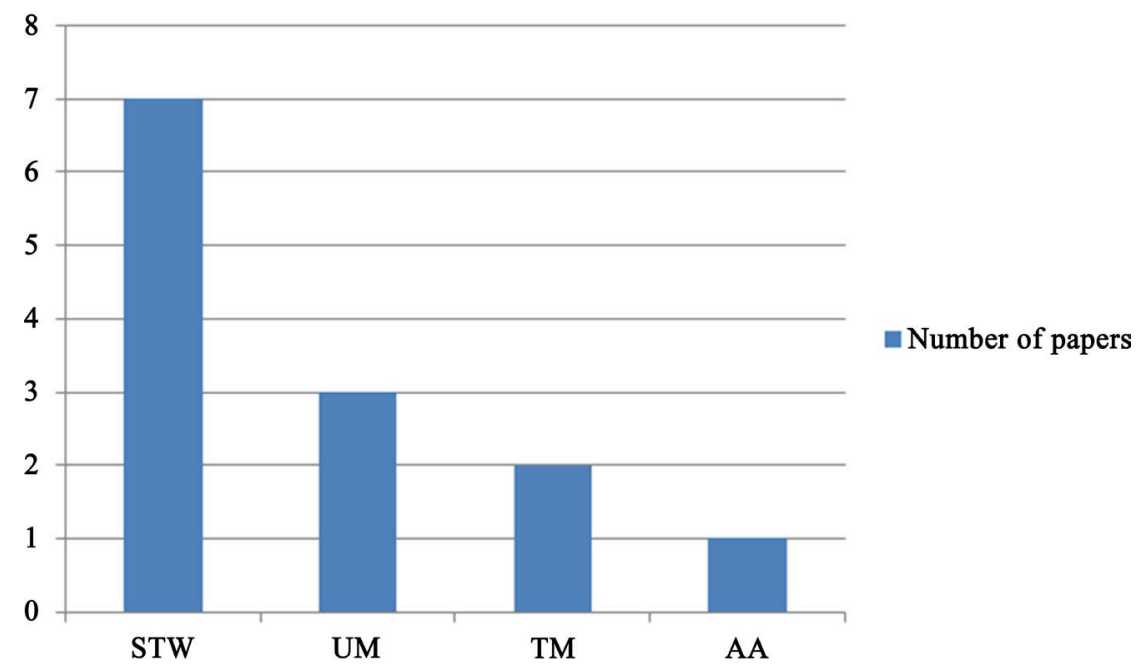

Figure 1. Statistical chart of MTPE literatures of science \& technology text in China. 
science and technology text machine translation post-editing papers can be reviewed as follows.

\subsection{Scientific and Technological Writings}

Most of the papers in which scientific and technological writings and reports are being used as source text to be translated with machine are translation practice reports. This kind of paper takes a scientific monograph as the source text, uses computer-aided translation software to translate the source text initially. What follows is the step of analyzing translation editing process, summarizing the errors in the machine translation, and finally putting forward specific translation strategies to guide the post-editing. For example, the article $A$ Report on the Translation of Machine Translation for Human Translators (Chapter One) (Wang, 2017) takes Machine Translation for Human Translators as an example to introduce the preparation work, difficulties encountered in the translation process and validation work for the edited excerpt of the book, and to illustrate the specific translation ideas and skills used in the translation process through typical cases. Fu Sihan adopts a similar approach, taking Translation and Web Localization as an example, and analyzes the post-editing process of the excerpt with Trados as a tool (Fu, 2017). Wang Yuhao adopts different model. The article takes the translation of patent abstracts as the research object instead of focusing on a single text. By analyzing the machine translation, it sums up the errors of 13 types of machine translation in three categories, such as omission of words and sentence components, and discusses how to improve the editing quality of such text style under the guidance of Skopos theory (Wang, 2017). Chen Qizu's paper also discusses the quality of post-editing. The article also adopts the model of MTPE. The source text of the article is the survey report of science and technology users and relevant science and technology text materials published online (Chen, 2014). This paper explores the errors in machine-translated texts with the criteria of non-literary translation summarized by Li Changshuan, and also discusses how to improve the quality of post-editing. The article $A$ Report on the Application of Post-editing on Defense Texts Translation (Xu, 2018) takes The Defense Industrial Base as the source text. Through the analysis of its machine translation, the article indicates that the post-editing process must consider the requirements of the project client and the expectations of the readers, and puts forward specific methods to improve the translation. The article A Study of Technical Texts Translation from the Perspective of Skopos Theory (Deng, 2013) also mentions that the purpose and pertinence of the translation must be considered in the post-editing process. Wang Juan's English-Chinese Translation Report on Curriculum Development is guided by Nida's functional equivalence theory (Wang, 2017). The source text Curriculum Development is compiled by summarizing the curriculum development experiences of two foreign universities. In the post-editing process, translation difficulties and strategies are pointed out, and the balance between translation quality and translation efficiency is dis- 
cussed.

The above papers mainly discuss the questions in two aspects: the practical process of post-editing and the evaluation of translation quality. For the question of the practical process of post-editing, most articles analyze the source text from the perspective of words, sentence patterns and chapters and point out the translation difficulties by analyzing a single source text such as a scientific and technological book. However, some of those don't have theoretical guidance, so the statement referred in these paper is not that reliable. For the question of the evaluation of translation quality, most articles adopts methods such as addition and omission to process the translation, sort out the differences between machine translation and manual translation, and put forward corresponding improvement strategies. However, most of these articles don't use CAT tools in the translation process, and the machine translation errors are not systematically analyzed. The CAT tools here refer to some translation software, like SDL Trados, which can help the translator summarize terms and MT errors, not Google website translation, as shown in Table 1 . Therefore, through the analysis, there are following questions: first, this kind of research model is relatively single, lacking in-depth research on different types of scientific and technological writings. Apart from Wang Yuhao's analysis of the translation of patent abstracts from a macro perspective, the rest of the articles mostly analyzes the specific writings of a text from a micro perspective and cannot conclude general conclusions from individual cases. Second, the translation strategy is not targeted, just focusing on the superficial meaning. Most of the translation strategies summarized in such articles are general strategies that are universally applicable, such as addition and omission. They do not dig deep into the characteristics of scientific and technological texts and put forward specific guiding strategies for the source

Table 1. Summary of the analysis of papers on scientific and technology writings.

Title
$\begin{gathered}\text { Theory } \\ \text { Guidance }\end{gathered} \begin{gathered}\text { CAT } \\ \text { Tools }\end{gathered} \begin{gathered}\text { Anstematic } \\ \text { MT Errors }\end{gathered}$
$\begin{gathered}\text { A Report on the Translation of Machine Translation for Human } \\ \text { Translators (Chapter One) }\end{gathered}$
$\begin{gathered}\text { On Post-editing of Machine Translation-A Report of Translating } \\ \text { Translation and Web Localization (excerpt) with Trados } \\ \text { A Report on C-E MTPE of Patent Abstract Translation } \\ \text { Translation Report on the Application of Machine Translation } \\ \text { and Post-editing on EST Translation }\end{gathered}$
A Report on the Application of Post-editing on Defense Texts
Translation


texts, thus providing limited guidance for translation practice. In addition, researchers are limited to the form of translation practice reports, and their application of translation theories is somewhat far-fetched.

\subsection{User Manual}

In the translation practice report category, manuals and guidelines for the use of science and technology are also taken as the research objects. The overall structure of the articles is similar to that of scientific and technological writings. The single manuals or guidelines for the use of science and technology are used as the source text, and the machine translation of the articles is analyzed to explore the errors in the translation in the post-editing process. For example, the article Practice Report on Post-editing Strategies for Chinese to English Machine Translation of a Software Usage Guide (Yang, 2018) analyzes the edited translation of the software usage guide, summarizes common errors in machine translation based on its own experience, and at the same time classifies the edited translation methods simply. Wang Yihan's article is the same model (Wang, 2018). The article makes a post-editing study of Localizing Apps: A Practical Guide for Translators and Translation Students and makes an error analysis of the machine-translated translation of the guide for the use of science and technology from morphology, syntax and discourse. A Report on the Chinese Translation of SmartCAT User Guide (Wang, 2018) also takes a similar model.

The three papers all focus on improving the quality of edited translation after translation. The overall structure of the articles is similar to that of scientific and technological writings, but there is difference in translation. None of the three articles proposes specific translation strategies for the translation of scientific and technological styles using manuals. The general analysis model of "machine translation + error analysis" is still adopted, which cannot guide the translation practice in a targeted way.

\subsection{Text Materials}

The citation of scientific and technological source texts and their research methods are diversified in articles of text materials. Although Guo Gaopan's analysis of pre-editing and post-editing of scientific and technological texts takes the focused text as source text, he uses empirical research methods to test and analyze scientific and technological materials instead of citing examples to prove that adding pre-editing and post-editing can get better translation script than using traditional direct translation by translators and also superior to translation that only processes mechanical translation (Guo \& Wang, 2017). Cui Qiliang's article also takes a unique approach in studying the quality of edited translation after translation. The study of English-Chinese machine translation of scientific and technological texts in this article does not focus on a single book or text, but analyzes the types of edited errors after translation by synthesizing various scientific and technological materials (Cui \& Li, 2015). Firstly, from the perspective of 
syntax, the article briefly analyzes the working principle of post-editing by constructing the syntax tree of English source text. Then, by analyzing the concrete examples of post-editing of scientific and technological texts, the article elaborates 11 types of post-editing errors, such as terminology translation errors, format errors, and part-of-speech judgment errors, etc., which also provide a macro guidance for post-editing quality (Guo \& Wang, 2017).

At present, there are only two papers on scientific and technological texts of written materials, but these two articles are very innovative. First of all, the paper is not limited to normative research. Guo Gaopan's article takes the lead in exploring the post-editing of scientific and technological texts by using empirical research methods, expanding the research methods. Secondly, it is not confined to a single text. Cui Qiliang's classification of errors in post-editing of scientific and technological translation is not obtained by analyzing a specific text, but by synthesizing various scientific and technological materials, and the types of errors pointed out are also very targeted, which has strong guiding significance for the translation of such styles in the future.

\subsection{Video and Audio}

At present, no one has been found to have done research on video and audio texts. The article A Feasibility Analysis of MTPE in Translation of Different Types of Documents (Tang, 2019) compares the machine translation of elevator installation instructions and subtitles of promotional films, and explains the processing methods of post-editing in different styles. However, the promotional films in this paper are promotional films for Hainan tourism, so this kind of content is not involved here.

A comprehensive analysis of the above documents shows that the researchers' research on post-editing of domestic scientific and technological texts mainly focuses on the evaluation of the quality of machine translation, and the source of the research texts are mostly single scientific and technological monographs or scientific and technological text materials. These documents are mostly translation practice reports, which may be difficult to seek new changes in form, but at the same time it also shows that researchers have not chosen the types of scientific and technological texts widely enough, especially the post-editing research on scientific and technological information, scientific and technological related conferences, audio and video and other texts still needs to be deepened.

Most of the above four types of researches focus on the quality and evaluation of translated texts edited after translation. Cui Yanqiu, on the other hand, has done further research on the cultivation of the translation ability of post-translation editors and translators from a unique perspective. Post-editing is comprehensive and should not be limited to the post-editing process. Information retrieval, project management, editing and typesetting should be included. Therefore, the article draws lessons from the teaching content of Nanyang Technological University's Translation Science and Technology course, discusses the post- 
editing ability of scientific and technological texts and the training of post-translation editors, puts forward a translation teaching mode that can be used for reference, and also explains the problems of improving the quality and efficiency of post-editing translation (Cui, 2017). This is the only relevant document on the teaching and training of editors and translators' abilities after the translation of scientific and technological texts. I believe more in-depth research will emerge in the future.

\section{Summary of Problems and Prospect of Trends}

MTPE is in the ascendant. Domestic scholars have paid more and more attention to relevant research in recent years. However, it can be seen from the literature review that there are still some problems in the post-editing research of domestic scientific and technological texts.

\subsection{Summary of Problems}

At present, there are still the following problems in MTPE of scientific and technological texts.

First of all, the perspective of post-editing research is relatively narrow, and most researches are repetitive. The source texts selected in the post-editing literature of domestic scientific and technological texts are mostly scientific and technological writings, and most of them are translation analysis of a scientific and technological monograph or a single material. The research focus is mostly on translation quality evaluation. Moreover, the model of analyzing machine translation errors and summarizing general strategies is adopted, and the problem of repetitive research has begun to emerge.

Secondly, the method of post-editing research is inflexible and empirical research is rare. International researches in this field mainly use empirical methods, and there have been abundant research results in recent years, but there are few research results in this field in China (Wang \& Jia, 2018). The research methods of domestic scientific and technological texts are mostly normative research, and the methods of optimizing translation quality are discussed. There are few empirical researches. In the future, the research angle can be appropriately turned to empirical research, so as to enrich the research data and make the research results more convincing. In addition, most of the current literatures focus on the macro analysis of the editing quality of a scientific and technological text after translation. After that, the classification of scientific and technological texts can be further refined, and the characteristics of different types of texts can be studied in a targeted way, which makes the research more instructive.

Thirdly, the form of post-editing research is relatively limited, and it has not entered the field of production-learning-research cooperation. "Production" here refers to the production of science and technology and the innovative practice of science and technology, "learning" refers to the training of post-translation editors of scientific and technological texts, while "research" refers to the rele- 
vant research on post-translation edited scientific and technological texts. The study on machine translation post-editing is mainly for the practice of machine translation post-editing (Zhang \& Feng, 2016). However, judging from the current research on post-editing of scientific and technological texts in China, the source texts chosen by researchers are mostly scientific and technological writings, and there is little research on scientific and technological fields that need urgent development and strong demand in the society, and there is little research on the training of specialized personnel for post-editing of scientific and technological texts. Future research can take the form of a combination of production-learning-research to make post-editing research of scientific and technological texts more targeted and better guide practice.

Although there are the above problems in the research on post-editing of scientific and technological texts in China, there are still some flaws. MTPE of scientific and technological texts is widely used and developing with each passing day. The author also puts forward some prospects here.

\subsection{Trend Outlook}

At present, with the continuous development of science and technology in our country, the demand for scientific and technological translation will become greater and greater. Post-editing of scientific and technological texts is also a new trend in the development of scientific and technological translation in the future. The author believes that future research on post-editing of scientific and technological texts can make more efforts in the following aspects.

First, expand the research field. The research on post-editing of scientific and technological texts is all-encompassing. Researchers can emancipate their minds and broaden their thinking. They can conduct research in a wide range of research fields, including the discussion on post-editing of scientific and technological texts, the research and development of relevant auxiliary translation tools for scientific and technological texts, the expansion and application of postediting in the scientific and technological industry, the post-editing ability of scientific and technological texts and the training of post-translation editors.

Second, enrich research methods. The research on post-editing of scientific and technological texts needs some theoretical guidance, especially the summarization of machine translation errors must be launched based on a specific theory. Researchers should not confine themselves to theoretical discussions, and it is also necessary to adopt empirical research methods and other methods. In addition, researchers can carry out targeted research on post-editing of scientific and technological texts according to different classifications in scientific and technological translation.

Third, the production, learning and research shall be cooperated. More CAT software needs to be added into the study so as to combine the research on the current development trends of the translation market. The social demand for scientific and technological text translation talents is strong, and post-editing is a 
major trend in the future translation development. Therefore, such research can adopt the form of combination of production, teaching and research, linking the training of scientific and technological post-editing talents in colleges and universities, post-editing research with the market and social demand, engaging in in in-depth cooperation, guiding practice with theoretical research, serving practice with personnel training, and forming a virtuous circle of production, teaching and research through summarizing practical experience, so as to achieve common development.

\section{Conclusion}

With the updating and iteration of science and technology in our country, MTPE is playing an increasingly important role in language services, and the society has more and more demands for post-editing of scientific and technological texts. However, looking at the current research on MTPE of scientific and technological texts in our country, we can find that researchers have not been deeply involved in this field, and there are still various problems in the existing research, such as the angle, method and form of research. In order to make the research in this field develop better and meet the increasing social demand, the future research on post-editing of scientific and technological texts should expand the research field and reasonably choose the source text type, change its research methods, find out the corresponding countermeasures, and use the "one-stop" form of production, teaching and research. This not only lays a theoretical foundation for providing more professional translation services, but also forms a virtuous circle and better promotes the overall development of post-editing of scientific and technological texts.

\section{Acknowledgements}

This work was supported by Project on Excellent ESP English Courses for Postgraduates in 2016 (No. JPK201605) and the Research Project on Teaching Reform of Shaanxi University of Science and Technology (No. 19Y068).

\section{Conflicts of Interest}

The authors declare no conflicts of interest regarding the publication of this paper.

\section{References}

Chen, Q. Z. (2014). Translation Report on the Application of Machine Translation and Post-Editing on EST Translation. Chongqing: Chongqing University.

Cui, Q. L., \& Li, W. (2015). Research on Types of Post-Editing Errors Based on English-Chinese Machine Translation of Scientific and Technological Texts. Chinese Science \& Technology Translators Journal, 28, 19-22.

Cui, Y. Q. (2017). Cultivation of Translation Skills-A Case Study of Science and Technology Course in Nanyang Technological University. Chinese Science \& Technology Translators Journal, 30, 23-25. 
Deng, H. M. (2013). A Study of Technical Texts Translation from the Perspective of Skopos Theory-A Report on Chinese Translation of Translation and Technology. Qingdao: Ocean University of China.

DePalma, D. A., \& Hegde, V. (2010). The Market for MT Post-Editing. Cambridge, MA: Common Sense Advisory.

Feng, Q. G., \& Cui, Q. L. (2016). Research Focuses and Trends in Post-Editing of Machine Translation. Shanghai Journal of Translators, No. 6, 67-74+89+94.

Fu, S. H. (2017). On Post-Editing of Machine Translation-A Report of Translating Translation and Web Localization (Excerpt) with Trados. Guangzhou: Guangdong University of Foreign Studies.

Fu, S. Y. (2016). The Critical Application of Machine Translation in Translation Practice-A Case of Search Engine Marketing, Inc. Translation Program. Qingdao: Qingdao University of Science and Technology.

Guo, G. P., \& Wang, Z. Y. (2017). Research on the Pre-Edit and Post-Edit of Machine Translation in Science and Technology Text Translation. Journal of Zhejiang International Studies University, No. 3, 76-83.

Liu, M. Q. (1998). English Varieties and Translation (p. 507). Beijing: China Foreign Translation Publishing Company.

Tang, Y. F. (2019). A Feasibility Analysis of $M T+P E$ in Translation of Different Types of Documents-A Case Study Based on a Lift Assembly Manual and a Video Script Translation Projects. Shanghai: Shanghai International Studies University.

Wang, F. (2017). A Report on the Translation of Machine Translation for Human Translators (Chapter One). Zhengzhou: Zhengzhou University.

Wang, J. (2017). An English Chinese Translation Report on "Curriculum Development" Based on Machine Translation. Chengdu: University of Electronic Science and Technology of China.

Wang, J. L. (2018). A Report on the Chinese Translation of Smart CAT User Guide. Yantai: Ludong University.

Wang, X. L., \& Jia, Y. F. (2018). Research Focus and Trends of Machine Translation Post-Editing. Journal of Hunan University (Social Sciences), No. 2, 82-87.

Wang, Y. H. (2017). A Report on C-E MTPE of Patent Abstract Translation. Nanjing: Southeast University.

Wang, Y. H. (2018). A Report on Machine Translation and Post-Editing of Localizing Apps: A Practical Guide for Translators and Translation Students (Excerpts). Guangzhou: Guangdong University of Foreign Studies.

Xu, J. (2018). A Report on the Application of Post-Editing on Defense Texts Translation-A Case of the Defense Industrial Base (Excerpts) Translation. Changsha: Hunan University.

Yang, M. (2018). Practice Report on Post-Editing Strategies for Chinese to English Machine Translation of a Software Usage Guide. Xi'an: Xi'an International Studies University.

Zhang, H. Y., \& Feng, Q. G. (2016). Pay Attention to Industry Development and Expand the Field of Translation Studies-Review on Post-Translation Editing of Machine Translation: Process and Application. Chinese Translation Journal, No. 2, 55-59. 